\title{
Response of complementary split ring resonators in composite stratified substrate integrated waveguide
}

J. M. Pérez-Escudero, Eduardo Jarauta, Francisco Falcone, and Miguel Beruete

Citation: Journal of Applied Physics 121, 194902 (2017); doi: 10.1063/1.4983709

View online: http://dx.doi.org/10.1063/1.4983709

View Table of Contents: http://aip.scitation.org/toc/jap/121/19

Published by the American Institute of Physics

\section{Articles you may be interested in}

Mode transition in cooperative metamaterials at terahertz frequencies

Journal of Applied Physics 121, 193101 (2017); 10.1063/1.4982758

Fano resonances in photonic crystal nanobeams side-coupled with nanobeam cavities

Journal of Applied Physics 121, 193102 (2017); 10.1063/1.4983450

Adaptable metasurface for dynamic anomalous reflection

Applied Physics Letters 110, 201904 (2017); 10.1063/1.4983782

Temperature dependence of photogalvanic effect in GaAs/AIGaAs two-dimensional electron gas at interband and intersubband excitation

Journal of Applied Physics 121, 193901 (2017); 10.1063/1.4983461

Ising computation based combinatorial optimization using spin-Hall effect (SHE) induced stochastic magnetization reversal

Journal of Applied Physics 121, 193902 (2017); 10.1063/1.4983636

Broadband locally resonant band gaps in periodic beam structures with embedded acoustic black holes Journal of Applied Physics 121, 194901 (2017); 10.1063/1.4983459

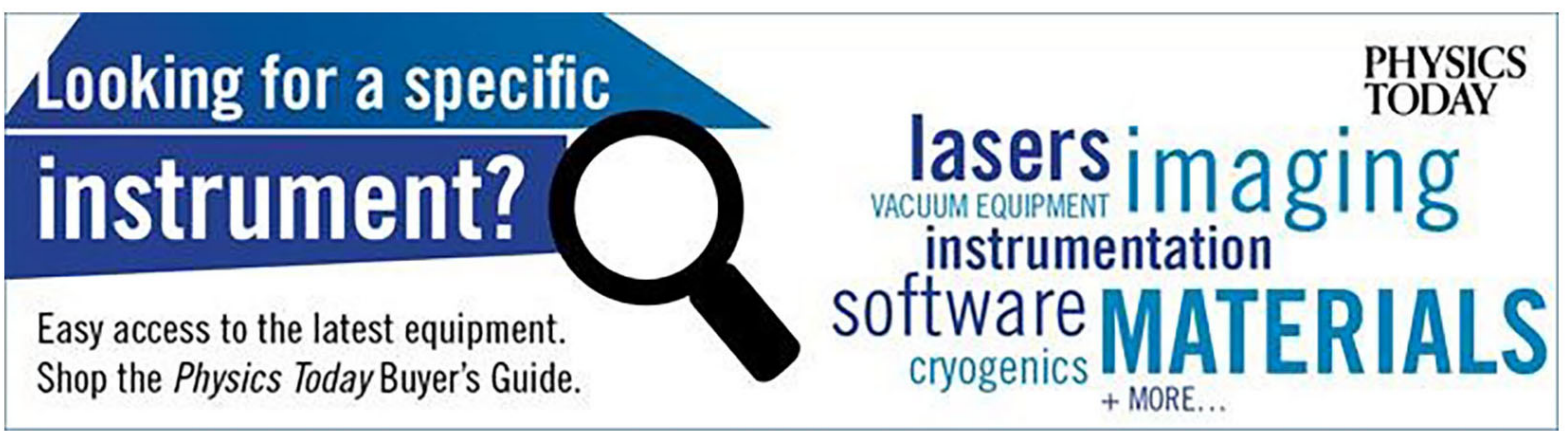




\title{
Response of complementary split ring resonators in composite stratified substrate integrated waveguide
}

\author{
J. M. Pérez-Escudero, ${ }^{1, a)}$ Eduardo Jarauta, ${ }^{2, b)}$ Francisco Falcone, ${ }^{2,3, c)}$ \\ and Miguel Beruete ${ }^{1,3, d)}$ \\ ${ }^{1}$ Antennas Group —TERALAB, Universidad Pública de Navarra, 31006 Pamplona, Spain \\ ${ }^{2}$ Electrical and Electronic Engineering Department, Universidad Pública de Navarra, 31006 Pamplona, Spain \\ ${ }^{3}$ Institute of Smart Cities, Public University of Navarre, 31006 Pamplona, Spain
}

(Received 7 April 2017; accepted 6 May 2017; published online 17 May 2017)

\begin{abstract}
In this work, the behavior of a Complementary Split Ring Resonator (CSRR) operating within the quasi-static resonance regime and embedded in a composite stratified Substrate Integrated Waveguide (SIW) with two different dielectric layers is analyzed. In these conditions, the propagating modes within the SIW combined with the imposed non-symmetrical structure lead to the excitation of the CSRR elements inside the SIW at difference with conventional excitation. Several cases of CSRR loaded SIW are proposed, and their electromagnetic field components as well as their frequency response are analyzed, providing insight into the CSRR resonance excitation. A test prototype has been designed, fabricated, and measured, showing a good agreement with simulation results and providing a new alternative for the implementation of compact frequency selective devices compatible with planar technology and without undesired radiation loss. Published by AIP Publishing.

[http://dx.doi.org/10.1063/1.4983709]
\end{abstract}

\section{INTRODUCTION}

The study of artificial electromagnetic media has attracted a great deal of attention of the scientific community within the last two decades. ${ }^{1}$ From the initial proposal of Kock $^{2}$ in the 1940s to employ artificial dielectrics to enhance radiation from a primary antenna, multiple alternatives have been described, such as Electromagnetic Bandgap structures in planar and volumetric configurations, ${ }^{3}$ or more recently, the implementation of multiple types of metamaterials and plasmonic media. ${ }^{4,5}$ A commonplace within these alternatives is the capability of engineering the electromagnetic response of the proposed equivalent medium by considering the inclusion of lattices of scatterers or loading elements within a host medium or the use of material modifications such as layered stratified media, anisotropic materials, or doped materials, to name a few.

A specific case of artificial media has been developed with the aid of small resonant elements, such as Split Ring Resonators, Open Ring Resonators, or Transmission Line based cells. Complementary versions in the case of Split Ring Resonators (SRRs) have also been proposed, in which application of Babinet's principle enables interchanging the role of electric and magnetic field excitation by interchanging metal/air elements within the initial circuit configuration. These elements, which in principle operate as equivalent resonators with small dimensions when compared to the source excitation wavelength, have been applied in a wide range of applications, such as filters, antennas, coupling devices, metasurfaces, or metalenses, to name a few. ${ }^{5}$

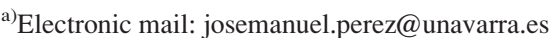

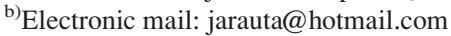

${ }^{c)}$ Electronic mail: francisco.falcone@unavarra.es

d)Electronic mail: miguel.beruete@unavarra.es
}

One of the main areas of interest is the implementation of enhanced devices in planar circuit technology in terms of size reduction and overall loss decrease, relevant in the development of a broad range of electronic products. Metamaterial inspired designs have been analyzed, given their potential miniaturization capabilities, applied to multiple planar transmission lines, from RF to the IR frequency range applied to optical communications and sensing/detection systems. ${ }^{6-8}$ In the specific case of microwave and millimeter wave bands, over the last years, there has been a significant increase in the use of substrate integrated waveguides (SIW) to implement devices in planar technology such as filters, combiners, and others. ${ }^{9,10}$ This growing interest is due to the high performance of the SIW line. First, this technology owns the advantages of the classical waveguides, such as high quality factor and low losses. Second, SIW also exhibits advantages of planar technology, such as low cost and easy compact integration. SIW are compatible with planar circuit technology, and their operation principle is similar to a dielectric loaded rectangular waveguide. The structure of a SIW is similar to a parallel plate waveguide, with the inclusion of two arrays of metallic cylinders, which connect top and bottom ground planes, resembling the lateral metallic walls of a metallic waveguide. To date, some works have exploited the combination of SIW technology with $\mathrm{SRR}^{11}$ and $\mathrm{CSRR}^{8,12}$ particles to develop miniaturized devices ${ }^{13-16}$ and filters. ${ }^{17,18}$ In these previous works, the CSRRs are placed on the ground plane layers of the SIW structure, leading to unavoidable radiation loss at resonance, ${ }^{19,20}$ which deteriorate filter performance.

In this paper, we overcome this drawback by embedding a CSRR particle inside the SIW structure. It will be demonstrated that the SIW must have an asymmetry so that the CSRR can be properly excited. We solve this issue by considering a composite substrate composed of two dielectric 


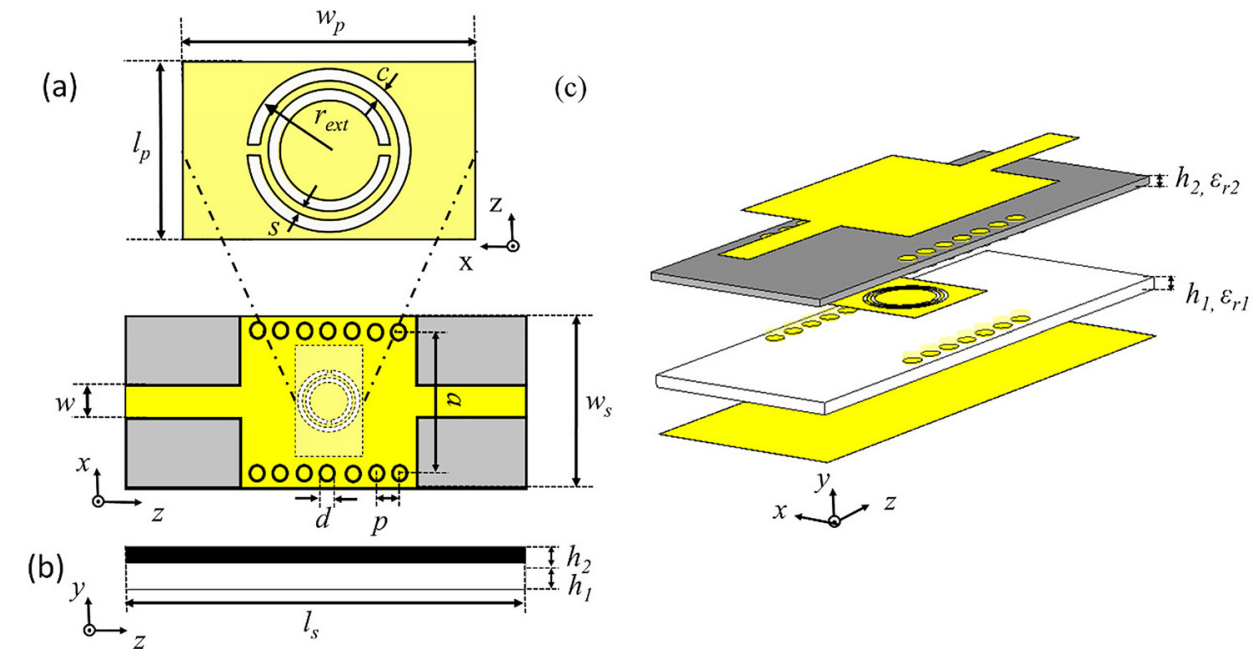

FIG. 1. Substrate integrated waveguide with a CSRR embedded on it. Top view and detail of the metallic layer with the CSRR etched on it (a), side view (b), and perspective representation showing all the layers (c).

slabs with different permittivity and height separated by a metallic layer where the CSRR is embedded. The paper is organized as follows: Section II provides a theoretical description of the excitation of CSRRs embedded within the SIW in terms of the considered modes. Section III provides an experimental validation by means of a planar SIW prototype, whereas Section IV presents the resulting conclusions.

\section{THEORETICAL ANALYSIS}

The structure proposed in this paper is a SIW composed of two slabs of dielectric material and a metallic patch with a CSRR etched on it inserted between both slabs. This waveguide is fed by a microstrip line placed on the top of the upper substrate. This kind of feeding is chosen because the Quasi Transversal Electromagnetic (QTEM) mode of the microstrip line is well suited to excite the fundamental mode of the SIW. This is given by the fact that the electric field of both modes is oriented in the same direction (i.e., strongly vertically polarized) and share, approximately, the same profile, giving rise to an efficient coupling between them. ${ }^{9}$ The bottom layer is covered by a metallic layer that acts as ground plane for both microstrip and SIW sections. A scheme of the structure is shown in Fig. 1.

Following the notation of the figure, we have the next parameters: $a=20 \mathrm{~mm}$ is the distance between the two rows of vias, $p=3 \mathrm{~mm}$ is the distance between vias, $d=2 \mathrm{~mm}$ is the via diameter, $l_{s}=48 \mathrm{~mm}$ is the length, $w_{s}=24 \mathrm{~mm}$ is the width of the structure, and $w=4 \mathrm{~mm}$ is the width of the feeding microstrip line. The parameters have been chosen following SIW operation and design lines described in Ref. 8. The CSRR has the next dimensions: $r_{\text {ext }}=3.9 \mathrm{~mm}$ is the external radius, $c=0.4 \mathrm{~mm}$ is the strip width, and $s=0.4 \mathrm{~mm}$ is the distance between rings, following CSRR embedded design rules, considering effective dielectric constant of the host medium. The CSRR is etched on a metallic patch with dimensions $l_{p}=9 \mathrm{~mm}$ and $w_{p}=14 \mathrm{~mm}$ (length and width, respectively). All the metal layers are made of copper with conductivity $\sigma=5.96 \times 10^{7} \mathrm{~S} / \mathrm{m}$ and thickness of $35 \mu \mathrm{m}$. Finally, the structure is composed of two dielectric slabs with height $h_{i}$ and relative permittivity $\varepsilon_{r i}$, where $i=1,2$ corresponds to the bottom and top slabs.
We divide the analysis into two different cases: uniform and composite filling. In the first case, the SIW is filled by dielectric slabs of the same permittivity $\varepsilon_{r l}=\varepsilon_{r l}=2.2$ (which corresponds to the commercial substrate Rogers RT5880) and height $h_{1}=h_{2}=0.79 \mathrm{~mm}$. In the second case, we replace the bottom substrate by a slab with permittivity $\varepsilon_{r l}=10.2$ (Rogers RO3010) and height $h_{l}=1.20 \mathrm{~mm}$, keeping the other slab as before.

First, we calculate the cutoff frequency of the first two modes in each case. For this analysis, we approximate the SIW by an equivalent rectangular waveguide of width $a_{e q}=a$ $-d .^{20}$ Then, the modal problem is solved using the transient solver of the full wave simulator CST Microwave Studio, setting the accuracy to $-60 \mathrm{~dB}$. A fine hexahedral mesh is applied with 40 lines per wavelength and lower mesh limit equal to 20 to map accurately the structure. Analyzing the port information, we obtain the results shown in Table I.

As shown below, the fundamental mode in the uniform case has a cutoff frequency of $5.32 \mathrm{GHz}$. In Fig. 2, we compare the modal distribution of a standard rectangular waveguide and a SIW, both with uniform filling. As shown there, the field pattern in both cases is very similar, almost indistinguishable, demonstrating the equivalence of both structures.

As it is known, due to inherent bianisotropic behavior, a CSRR operating at the quasistatic resonance can be excited by either an axial electric field or a tangent magnetic field perpendicular to the small strips of the rings, provided that the excitation is asymmetric with respect to the plane of the CSRR. ${ }^{17,18}$ As observed in the figure, both the electric and magnetic field components of the fundamental mode of a uniformly filled SIW (and also in the case of a rectangular

TABLE I. Information of the type and cutoff frequency of the modes of the two studied cases.

\begin{tabular}{lccc}
\hline \hline Case & Mode & Type & $f_{\mathrm{c}}$ \\
\hline Homog. & 1 & TE & 5.32 \\
& 2 & TE & 10.64 \\
Inhomog. & 1 & Hybrid & 4.38 \\
& 2 & Hybrid & 6.68 \\
\hline \hline
\end{tabular}

$f_{c}$ is the cutoff frequency of the mode in $\mathrm{GH} z$. 
(a)

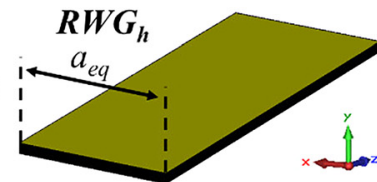

(b)

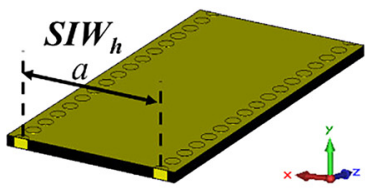

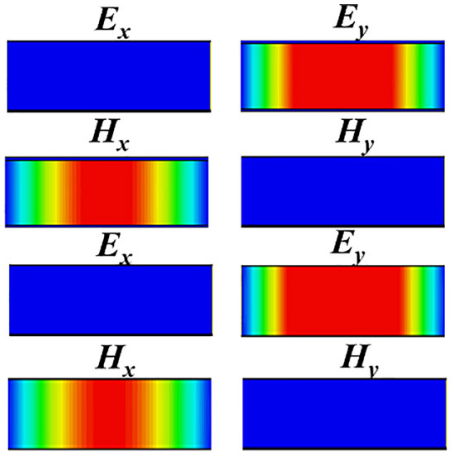

0
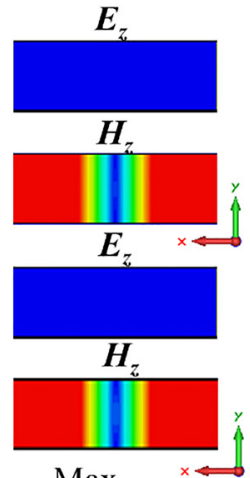

$\operatorname{Max}$
FIG. 2. Modal distribution showing the magnitude of all field components in the $x y$-plane $(\mathrm{z}=0)$ for a rectangular waveguide with uniform dielectric filling (a) and a SIW with uniform dielectric filling (b). The maximum value (Max) of the colorbar is $6000 \mathrm{~V} / \mathrm{m}$ for $E_{y}, 600 \mathrm{~V} / \mathrm{m}$ for $E_{x}, E_{z}$, and $15 \mathrm{~A} / \mathrm{m}$ for $H_{x}, H_{y}, H_{z}$ waveguide) have translational symmetry, i.e., are invariant along the $y$-axis, and hence, all field components are exactly identical above and below the CSRR. This means that, as shown in Fig. 4, it is impossible to excite the CSRR by using a uniform dielectric filling, regardless of the position of the CSRR along the vertical axis or the orientation of the CSRR (see the Appendix for completeness). For this reason, the spectral response with a CSRR is not modified compared to a SIW without CSRR, as shown in Fig. 5 (dashed blue lines). To ensure that this is not due to having the CSRR resonance below the cutoff frequency of the waveguide, we performed a numerical study with an artificial waveguide of mutually perpendicular electric and magnetic planes, whose fundamental mode is of the TEM type (see results within the Appendix).

From the previous discussion, it is clear that it is necessary to have an asymmetric field distribution to excite the CSRR. This can be accomplished with the composite filling mentioned above. As shown in Table I, the fundamental mode has a cutoff frequency of $4.38 \mathrm{GHz}$ and the second mode has a cutoff frequency of $6.68 \mathrm{GHz}$. To design the CSRR, we inserted it inside an artificial waveguide with mutually perpendicular electric and magnetic planes whose cutoff frequency is zero (note that due to the composite filling, the fundamental mode is QTEM, not TEM, but the cutoff frequency is still equal to zero). With this analysis, we obtained a resonance frequency of $5.59 \mathrm{GHz}$ (see the Appendix), i.e., inside the monomode operation range of the SIW.

Concentrating now on the SIW results of Fig. 3, we observe that due to the composite filling, both $E_{x}$ and $E_{z}$ components are no longer null but, in any case, they are still negligible compared with the dominant $E_{y}$ component (the scale has been reduced by an order of magnitude to visualize them). Furthermore, $E_{y}$ is obviously asymmetric and mainly concentrated on the slab of lower $\varepsilon_{r}$. As shown in Fig. 4 (composite), this configuration fulfills the conditions to excite the CSRR, giving rise to a dip in the spectral response at $5.59 \mathrm{GHz}$ (see solid red lines in Fig. 5).

\section{EXPERIMENTAL VALIDATION}

To corroborate the previous analysis, the composite circuit was fabricated by using a LPKF ProtoMat H100. In Fig. 6 is shown the top view and side view of the fabricated device. It was characterized using an Agilent 8722ES S-Parameter Vector Network Analyzer.

Figure 7 shows a comparison between the simulated and measured transmission and reflection coefficients. Aside from a small frequency shift towards lower frequencies in the measurements, there is a good agreement between the numerical and experimental results. The slight disagreement might be due to fabrication tolerances inherent with the employed mechanical fabrication process. Nonetheless, it is clear that the CSRR can be excited in this asymmetric configuration, giving rise to a notch near $5.2 \mathrm{GHz}$ in the experimental results. Furthermore, the circuit is operating as a bandpass filter for the rest of frequencies above the cutoff frequency. It is noteworthy that the losses of the filter are very low, because the radiation losses are avoided, thanks to embedding the CSRR between two ground planes. (a)

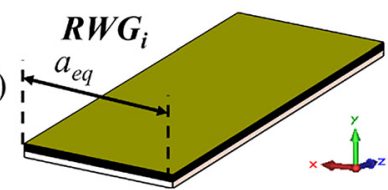

(b)

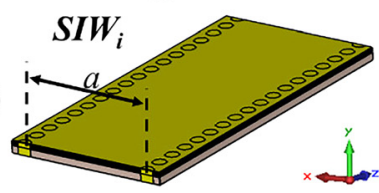

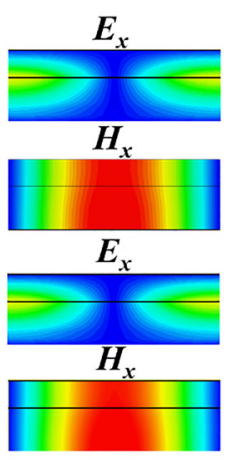

0

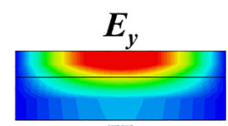

$H_{p}$

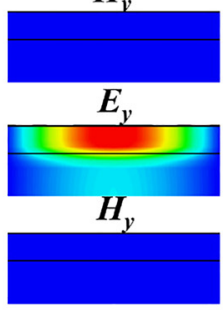

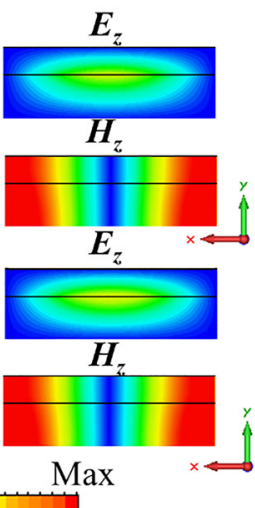

FIG. 3. Modal distribution showing the magnitude of all field components in the $x y$-plane $(\mathrm{z}=0)$ for a rectangular waveguide with composite dielectric filling (a) and a SIW with composite dielectric filling (b). The maximum value (Max) of the colorbar is $6000 \mathrm{~V} /$ $\mathrm{m}$ for $E_{y}, 600 \mathrm{~V} / \mathrm{m}$ for $E_{x}, E_{z}$, and $15 \mathrm{~A} /$ m for $H_{x}, H_{y}, H_{z}$ 


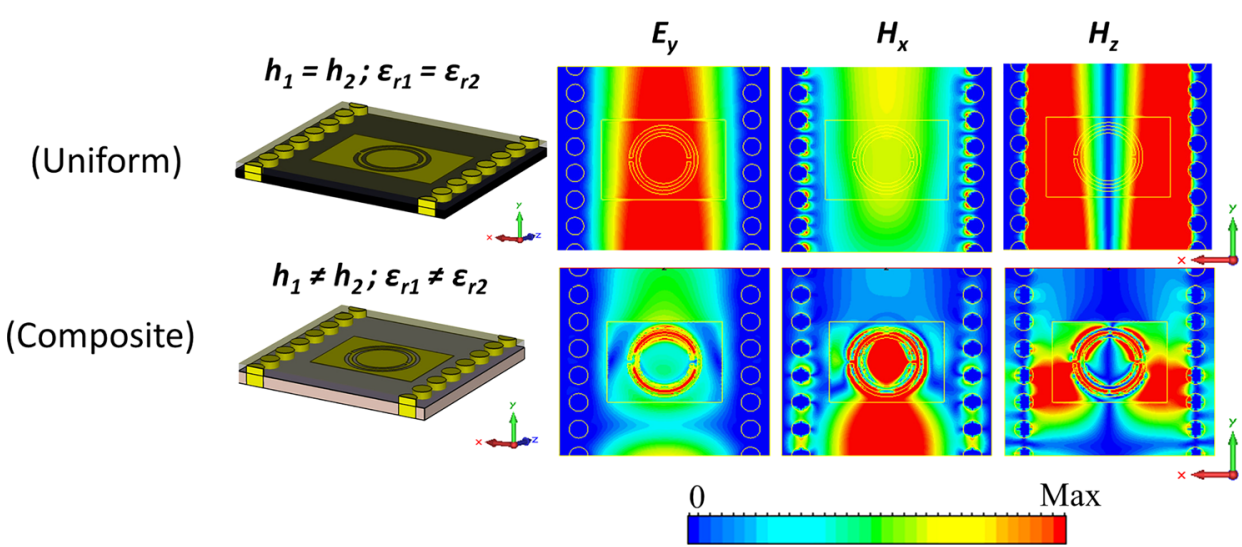

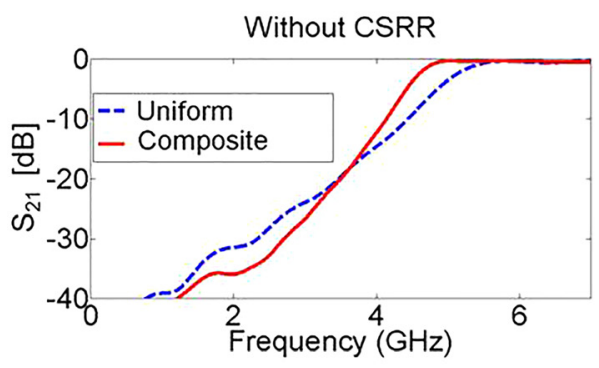

(a)

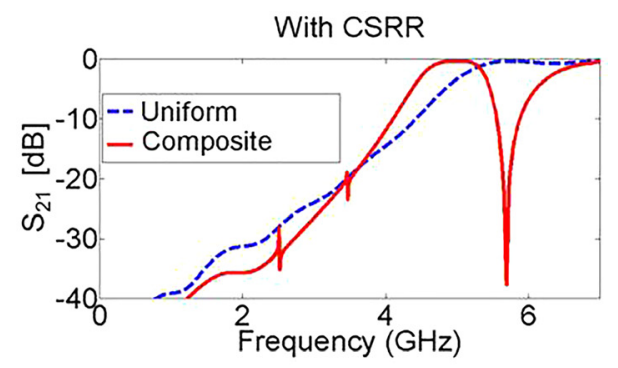

(b)

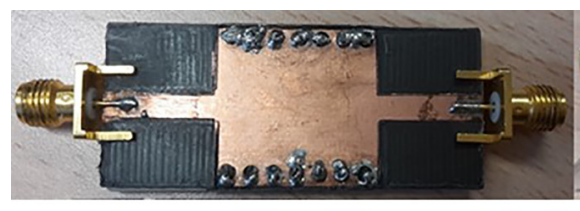

(a)

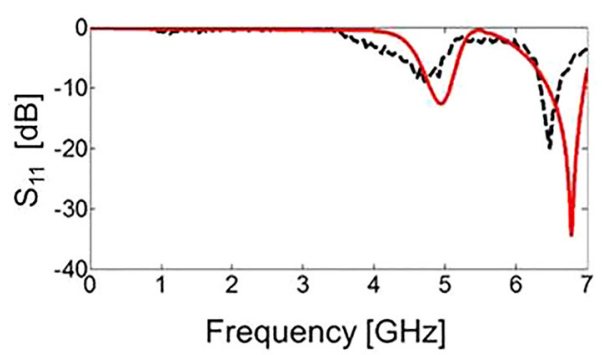

(a)

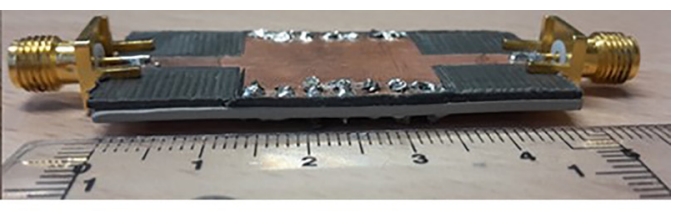

(b)

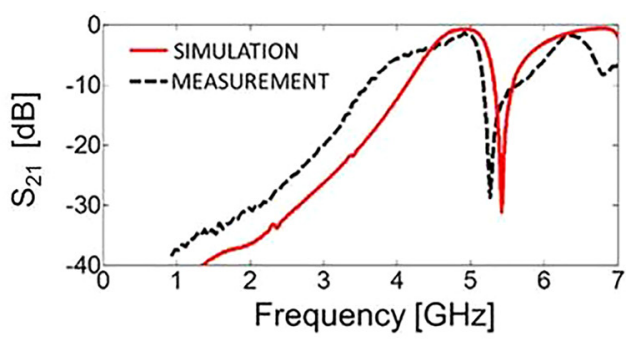

(b)
FIG. 6. Top view (a) and side view (b) of the fabricated prototype.
FIG. 4. Top view of the magnitude of the E-field and H-field in a SIW with a CSRR: uniform SIW (top) and composite SIW (bottom). The maximum value (Max) of the colorbar is $6000 \mathrm{~V} /$ $\mathrm{m}$ for $E_{y}$ and $15 \mathrm{~A} / \mathrm{m}$ for $H_{x}, H_{z}$.
FIG. 5. Simulated $S_{21}$ without (a) and with (b) CSRR for a uniformly filled SIW (dashed blue lines) and a composite filled SIW (solid red lines).
FIG. 7. Simulated (solid red line) and measured (dashed black line) $\mathrm{S}_{11}$ (a) and $\mathrm{S}_{21}(\mathrm{~b})$.

\section{CONCLUSIONS}

In this work, we have performed a detailed analysis of a SIW loaded with a CSRR grabbed in a copper layer and inserted inside. As it has been shown, the necessary condition to excite the quasistatic CSRR resonance is to have an asymmetrical field distribution along the $y$ axis. This condition cannot be fulfilled in a SIW with a uniform filling, due to the translational invariance of the fields. Therefore, a composite filling with two different dielectric layers is necessary. The performance of the composite prototype has been evaluated experimentally at microwaves, with a very good agreement with the numerical results, corroborating the theoretical explanation. Due to its geometry, the filter devised avoids the radiation losses, improving the overall performance compared with other designs existing in the literature. Hence, the use of the CSRR within a SIW opens a new future on the development of new applications and devices, avoiding radiation losses.

\section{ACKNOWLEDGMENTS}

This work was sponsored by the Spanish Government under Contract No. TEC2014-51902-C2-2-R. M.B. was sponsored by the Spanish Government via RYC-2011-08221.

\section{APPENDIX: NUMERICAL STUDY OF THE CSRR EXCITATION IN AN ARTIFICIAL TEM WAVEGUIDE}

In this Appendix, we perform several numerical studies of a CSRR embedded in an artificial waveguide with mutually 
(a)

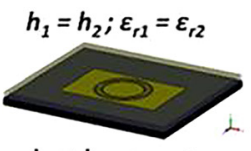

(b)
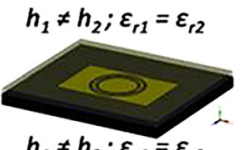

(c)
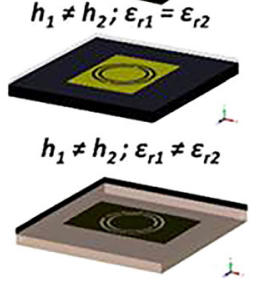

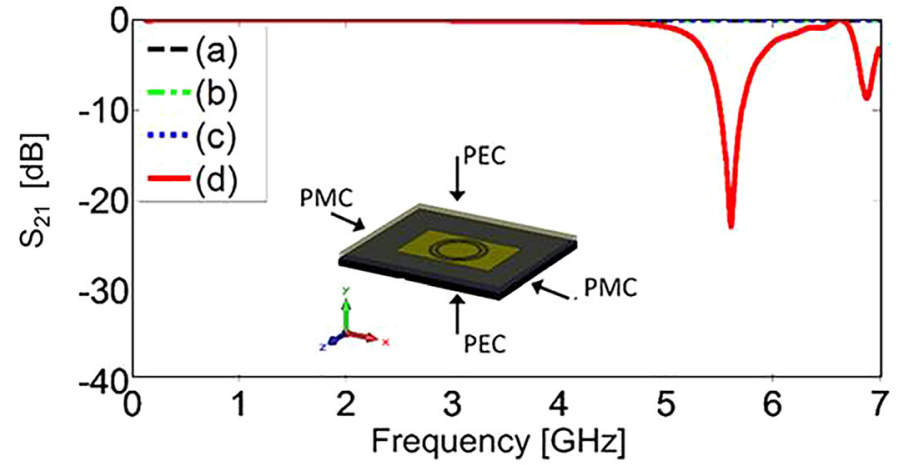

FIG. 8. Simulated S parameters for the cases: uniform SIW and CSRR at $y=0 \mathrm{~mm}$ (a), $y=0.4 \mathrm{~mm}$ (b), $y=0.4$ $\mathrm{mm}$ and rotated $90^{\circ}$ along $y(\mathrm{c})$, and composite SIW (d). (Inset) Artificial transmission line. perpendicular electric and magnetic walls. We begin the analysis with a uniformly filled artificial waveguide (see inset of Fig. 8) that supports a TEM mode to prove that the CSRR is not excited in any configuration, due to the symmetry of the fields. We consider a CSRR embedded inside a uniform dielectric filling with permittivity $\varepsilon_{r}=2.2$ and several configurations: (a) CSRR in the middle, i.e., at $y=0 \mathrm{~mm}$; (b) CSRR at $y=0.4 \mathrm{~mm}$, and (c) CSRR at $y=0.4 \mathrm{~mm}$ and rotated $90^{\circ}$ along the $y$ axis. As shown in Fig. 8, none of these cases show a dip in the spectral response, showing that the CSRR cannot be excited by an axial electric field [cases (a) and (b)] or a magnetic field [case (c)], due to the symmetry of the fields. This proves that the absence of dips in the spectral response of the uniform case discussed in the main text is not due to having the CSRR resonance below the cutoff frequency of the waveguide. In contrast, when we consider a composite filling [Fig. 8(d)] like the one discussed in the main text, we obtain a clear dip in the spectral response at $5.59 \mathrm{GHz}$ which is the frequency mentioned in the main text.

${ }^{1}$ R. E. Collin, Field Theory of Guided Waves (McGraw-Hill, New York, NY, 1960).

${ }^{2}$ W. E. Kock, Proc. IRE 34, 828 (1946).

${ }^{3}$ J. D. Joannopoulos, R. D. Meade, and J. N. Winn, Photonic Crystals: Molding the Flow of Light (Princeton University Press, 1995).

${ }^{4}$ S. A. Maier, Plasmonics: Fundamentals and Applications (Springer, New York, 2007).
${ }^{5}$ R. Marqués, F. Martín, and M. Sorolla, Metamaterials with Negative Parameters: Theory, Design and Microwave Applications (John Wiley \& Sons, 2008).

${ }^{6}$ N. Engheta and R. W. Ziolkowski, Metamaterials: Physics and Engineering Explorations (John Wiley \& Sons, Hoboken, New Jersey, 2006).

${ }^{7} \mathrm{C}$. Caloz and T. Itoh, Electromagnetic Metamaterials: Transmission Line Theory and Microwave Applications (John Wiley \& Sons, 2005).

${ }^{8}$ D. Deslandes and K. Wu, IEEE Trans. Microwave Theory Tech. 51, 593 (2003).

${ }^{9}$ X.-P. Chen, K. Wu, and Z.-L. Li, IEEE Trans. Microwave Theory Tech. 55, 2569 (2007).

${ }^{10}$ J. B. Pendry, A. J. Holden, D. J. Robbins, and W. J. Stewart, IEEE Trans. Microwave Theory Tech. 47, 2075 (1999).

${ }^{11}$ F. Falcone, T. Lopetegi, and J. Baena, IEEE Microwave Wireless Compon. Lett. 14, 280 (2004)

${ }^{12}$ J. D. Baena, J. Bonache, F. Martin, R. M. Sillero, F. Falcone, T. Lopetegi, M. A. G. Laso, J. Garcia-Garcia, I. Gil, M. F. Portillo, and M. Sorolla, IEEE Trans. Microwave Theory Tech. 53, 1451 (2005).

${ }^{13} \mathrm{M}$. Gil, J. Bonache, and F. Martin, in IEEE MTT-S International Microwave Symposium Digest (2008), p. 323.

${ }^{14}$ K. Dong, J. Mo, Y. He, and Z. Ma, Microw. Opt. Technol. Lett. 58, 2406 (2016).

${ }^{15}$ K. Wang, H. Tang, R. Wu, C. Yu, J. Zhang, and X. Wang, Microwave Opt. Technol. Lett. 58, 2704 (2016).

${ }^{16}$ D. E. Senior, X. Cheng, M. Machado, and Y.-K. Yoon, in IEEE Antennas and Propagation Society International Symposium (2010), p. 1.

${ }^{17}$ W. Park and S. Lim, in 41 st European Microwave Conference (2011), pp. 740-743.

${ }^{18}$ L.-S. W. Qiao-Li Zhang, W.-Y. Yin, and S. He, in 2010 Asia-Pacific Microwave Conference (2010), Vol. 20, p. 1875.

${ }^{19}$ Y. D. Dong, T. Yang, and T. Itoh, IEEE Trans. Microwave Theory Tech. 57, 2211 (2009).

${ }^{20}$ W. Che, K. Deng, D. Wang, and Y. L. Chow, IET Microwave Antennas Propag. 626 SP, 35 (2008). 